\title{
Sequential Generalized Transforms on Function Space
}

\author{
Jae Gil Choi, Hyun Soo Chung, and Seung Jun Chang \\ Department of Mathematics, Dankook University, Cheonan 330-714, Republic of Korea \\ Correspondence should be addressed to Seung Jun Chang; sejchang@dankook.ac.kr
}

Received 20 August 2013; Accepted 2 November 2013

Academic Editor: Douglas Anderson

Copyright (C) 2013 Jae Gil Choi et al. This is an open access article distributed under the Creative Commons Attribution License, which permits unrestricted use, distribution, and reproduction in any medium, provided the original work is properly cited.

We define two sequential transforms on a function space $C_{a, b}[0, T]$ induced by generalized Brownian motion process. We then establish the existence of the sequential transforms for functionals in a Banach algebra of functionals on $C_{a, b}[0, T]$. We also establish that any one of these transforms acts like an inverse transform of the other transform. Finally, we give some remarks about certain relations between our sequential transforms and other well-known transforms on $C_{a, b}[0, T]$.

\section{Introduction and Preliminaries}

Let $C_{0}[0, T]$ denote one-parameter Wiener space; that is, the space of all real-valued continuous functions $x(t)$ on $[0, T]$ with $x(0)=0$. The study of the Fourier-Wiener transform of functionals on $C_{0}[0, T]$ was initiated by Cameron and Martin [1-3]. This transform and its properties are similar in many respects to the ordinary Fourier function transform. Since then, many transforms which were somewhat analogous to the Fourier-Wiener transform have been defined and developed in the literature. There are two well-known transforms on $C_{0}[0, T]$. One of them is the analytic Fourier-Feynman transform [4-6] and the other is the integral transform [710]. Each of the transforms on Wiener space has an inverse transform. For an elementary survey, see [11].

In [12-16], the authors studied the generalized analytic Fourier-Feynman transform and the generalized integral transform for functionals defined on a more general function space $C_{a, b}[0, T]$. The function space $C_{a, b}[0, T]$, induced by generalized Brownian motion process, was introduced by Yeh $[17,18]$ and was used extensively by Chang and Chung [19]. The Wiener process used in [1-10] is stationary in time and is free of drift, while the stochastic process used in this paper, as well as in $[12-17,19]$, is nonstationary in time and is subject to a drift $a(t)$. In case $a(t) \equiv 0$ and $b(t)=t$ on $[0, T]$, the general function space $C_{a, b}[0, T]$ reduces to the Wiener space $C_{0}[0, T]$ and so most of the results in $[4-6,9]$ follow immediately from the results in $[12,13,15,16]$.
However, the existence of an inverse transform of each of the two generalized transforms on $C_{a, b}[0, T]$ has not yet been established. It is a critical point that the generalized transforms on $C_{a, b}[0, T]$ are essentially different from the transforms on Wiener space $C_{0}[0, T]$. The main purpose of this paper is to define a transform on $C_{a, b}[0, T]$ which has an inverse transform.

In this paper, we define two sequential transforms on the function space $C_{a, b}[0, T]$. To do this, we investigate a representation for sample paths of the generalized Brownian motion process and introduce the concept of the $\mathscr{G}$-scontinuity for functionals on $C_{a, b}[0, T]$. We then proceed to establish the existence of the sequential transforms for functionals in a Banach algebra $\mathscr{F}^{*}\left(C_{a, b}[0, T]\right)$ of functionals on $C_{a, b}[0, T]$. Next, we establish that any one of these transforms acts like an inverse transform of the other transform. Finally, we examine certain aspects of the generalized analytic Fourier-Feynman transform, the generalized integral transform, and the sequential transforms.

We briefly list some of the preliminaries from $[12,13,17]$ that we will need in order to establish the results in this paper.

Let $a(t)$ be an absolutely continuous real-valued function on $[0, T]$ with $a(0)=0, a^{\prime}(t) \in L^{2}[0, T]$, and let $b(t)$ be a strictly increasing, continuously differentiable real-valued function with $b(0)=0$ and $b^{\prime}(t)>0$ for each $t \in[0, T]$. The generalized Brownian motion process $Y$ determined by $a(t)$ and $b(t)$ is a Gaussian process with mean function $a(t)$ 
and covariance function $r(s, t)=\min \{b(s), b(t)\}$. For more details, see [12, 13]. By Theorem 14.2 in [18], the probability measure $\mu$ induced by $Y$, taking a separable version, is supported by $C_{a, b}[0, T]$ (which is equivalent to the Banach space of continuous functions $x$ on $[0, T]$ with $x(0)=0$ under the sup norm). Hence, $\left(C_{a, b}[0, T], \mathscr{B}\left(C_{a, b}[0, T]\right), \mu\right)$ is the function space induced by $Y$ where $\mathscr{B}\left(C_{a, b}[0, T]\right)$ is the Borel $\sigma$-algebra of $C_{a, b}[0, T]$. We then complete this function space to obtain $\left(C_{a, b}[0, T], \mathscr{W}\left(C_{a, b}[0, T]\right), \mu\right)$, where $\mathscr{W}\left(C_{a, b}[0, T]\right)$ is the set of all Wiener measurable subsets of $C_{a, b}[0, T]$.

A subset $B$ of $C_{a, b}[0, T]$ is said to be scale-invariant measurable provided $\rho B$ is $\mathscr{W}\left(C_{a, b}[0, T]\right)$-measurable for all $\rho>0$, and a scale-invariant measurable set $N$ is said to be a scale-invariant null set provided $\mu(\rho N)=0$ for all $\rho>0$. A property that holds except on a scale-invariant null set is said to hold scale-invariant almost everywhere (s-a.e.). A functional $F$ is said to be scale-invariant measurable provided $F$ is defined on a scale-invariant measurable set, and $F(\rho \cdot)$ is $\mathscr{W}\left(C_{a, b}[0, T]\right)$-measurable for every $\rho>0$. If two functionals $F$ and $G$ defined on $C_{a, b}[0, T]$ are equal s-a.e., we write $F \approx G$.

Let $L_{a, b}^{2}[0, T]$ be the space of functions on $[0, T]$ which are Lebesgue measurable and square integrable with respect to the Lebesgue-Stieltjes measures on $[0, T]$ induced by $a(\cdot)$ and $b(\cdot)$; that is,

$$
\begin{aligned}
& L_{a, b}^{2}[0, T] \\
& \quad=\left\{v: \int_{0}^{T} v^{2}(s) d b(s)<+\infty, \int_{0}^{T} v^{2}(s) d|a|(s)<+\infty\right\},
\end{aligned}
$$

where $|a|(\cdot)$ is the total variation function of $a(\cdot)$. Then, $\left(L_{a, b}^{2}[0, T],\|\cdot\|_{a, b}\right)$ is a separable Hilbert space with inner product defined by

$$
(u, v)_{a, b}=\int_{0}^{T} u(t) v(t) d[b(t)+|a|(t)] .
$$

Note that $\|u\|_{a, b}=\sqrt{(u, u)_{a, b}}=0$ if and only if $u(t)=0$ a.e. on $[0, T]$. Also note that all functions of bounded variation on $[0, T]$ are elements of $L_{a, b}^{2}[0, T]$. If $a(t) \equiv 0$ and $b(t)=t$, then $L_{a, b}^{2}[0, T]=L^{2}[0, T]$. In fact,

$$
\begin{aligned}
\left(L_{a, b}^{2}[0, T],\|\cdot\|_{a, b}\right) & \subset\left(L_{0, b}^{2}[0, T],\|\cdot\|_{0, b}\right) \\
& =\left(L^{2}[0, T],\|\cdot\|_{2}\right)
\end{aligned}
$$

because the norms $\|\cdot\|_{0, b}$ and $\|\cdot\|_{2}$ are equivalent.

Let

$$
\begin{aligned}
C_{a, b}^{\prime}[0, T] & =\left\{w \in C_{a, b}[0, T]: w(t)\right. \\
& \left.=\int_{0}^{t} z(s) d b(s) \text { for some } z \in L_{a, b}^{2}[0, T]\right\} .
\end{aligned}
$$

For $w \in C_{a, b}^{\prime}[0, T]$, with $w(t)=\int_{0}^{t} z(s) d b(s)$ for $t \in[0, T]$, let $D: C_{a, b}^{\prime}[0, T] \rightarrow L_{a, b}^{2}[0, T]$ be defined by the formula

$$
D w(t)=z(t)=\frac{w^{\prime}(t)}{b^{\prime}(t)} .
$$

Then $C_{a, b}^{\prime} \equiv C_{a, b}^{\prime}[0, T]$ with inner product

$$
\begin{aligned}
\left(w_{1}, w_{2}\right)_{C_{a, b}^{\prime}} & =\int_{0}^{T} D w_{1}(t) D w_{2}(t) d b(t) \\
& =\int_{0}^{T} z_{1}(t) z_{2}(t) d b(t)=\left(z_{1}, z_{2}\right)_{0, b}
\end{aligned}
$$

is a separable Hilbert space.

Note that the two separable Hilbert spaces $L_{a, b}^{2}[0, T]$ and $C_{a, b}^{\prime}[0, T]$ are homeomorphic under the linear operator given by (5). The inverse operator of $D$ is given by

$$
\left(D^{-1} z\right)(t)=\int_{0}^{t} z(s) d b(s) .
$$

Recall that above, as well as in papers [12-16], we require that $a:[0, T] \rightarrow \mathbb{R}$ is an absolutely continuous function with $a(0)=0$ and with $\int_{0}^{T}\left|a^{\prime}(t)\right|^{2} d t<+\infty$. Our conditions on $b:[0, T] \rightarrow \mathbb{R}$ imply that $\delta<b^{\prime}(t)<\Delta$ for some positive real numbers $\delta$ and $\Delta$, and $b^{\prime}(t)$ is continuous on $[0, T]$. Hence, we have

$$
\int_{0}^{T}\left|a^{\prime}(t)\right|^{2} d b(t)=\int_{0}^{T}\left|a^{\prime}(t)\right|^{2} b^{\prime}(t) d t<+\infty .
$$

But we cannot ensure that

$$
\int_{0}^{T}\left|a^{\prime}(t)\right|^{2} d|a|(t)<+\infty
$$

under current conditions. Note that the function $a(t)=t^{2 / 3}$, $0 \leq t \leq T$, does not satisfy condition (9) even though its derivative is an element of $L^{2}[0, T]$.

In this paper, we add the requirement (9). Then we obtain the following lemma.

Lemma 1. The function $a:[0, T] \rightarrow \mathbb{R}$ satisfies the requirement (9) if and only if $a$ is an element of $C_{a, b}^{\prime}[0, T]$.

Under the requirement (9), we observe that for each $z \in$ $L_{a, b}^{2}[0, T]$,

$$
\int_{0}^{T} z(t) d a(t)=\int_{0}^{T} D w(t) D a(t) d b(t)=(w, a)_{C_{a, b}^{\prime}},
$$

where $w(t)=\int_{0}^{t} z(s) d b(s)$ for $t \in[0, T]$.

The following integration formula is used several times in this paper:

$$
\int_{\mathbb{R}} \exp \left\{-\alpha u^{2}+\beta u\right\} d u=\sqrt{\frac{\pi}{\alpha}} \exp \left\{\frac{\beta^{2}}{4 \alpha}\right\}
$$

for complex numbers $\alpha$ and $\beta$ with $\operatorname{Re}(\alpha)>0$.

\section{A Representation for Paths $x$ in $C_{a, b}[0, T]$}

In this section, we investigate a representation for paths $x$ in $C_{a, b}[0, T]$. To do this, we first define a Paley-WienerZygmund (PWZ) type stochastic integral. 
Let $\left\{g_{1}, g_{2}, \ldots\right\}$ be a complete orthonormal set of functions in the separable Hilbert space $C_{a, b}^{\prime}[0, T]$, such that for each $j=1,2, \ldots, D g_{j}(t)=\alpha_{j}(t)$ is of bounded variation on $[0, T]$. Then, for each $w \in C_{a, b}^{\prime}[0, T]$ with $D w=z \in$ $L_{a, b}^{2}[0, T]$, we can write

$$
w(t)=\sum_{j=1}^{\infty}\left(w, g_{j}\right)_{C_{a, b}^{\prime}} g_{j}(t)=\sum_{j=1}^{\infty}\left(z, \alpha_{j}\right)_{0, b} g_{j}(t)
$$

on $[0, T]$.

For each $w=D^{-1} z \in C_{a, b}^{\prime}[0, T]$, the PWZ type stochastic integral $(w, x)_{C_{a, b}^{\prime}}^{\sim}$ is defined by the formula

$$
\begin{aligned}
(w, x)_{\tilde{C}_{a, b}^{\prime}}^{\sim} & =\lim _{n \rightarrow \infty} \int_{0}^{T} \sum_{j=1}^{n}\left(w, g_{j}\right)_{C_{a, b}^{\prime}} D g_{j}(t) d x(t) \\
& =\lim _{n \rightarrow \infty} \int_{0}^{T} \sum_{j=1}^{n}\left(z, \alpha_{j}\right)_{0, b} \alpha_{j}(t) d x(t)
\end{aligned}
$$

for all $x \in C_{a, b}[0, T]$ for which the limit exists.

The following are some basic properties of the PWZ type stochastic integral. They are nontrivial, but straightforward to prove.

(1) For each $w \in C_{a, b}^{\prime}[0, T]$, the PWZ type stochastic integral $(w, x)_{C_{a, b}^{\prime}}^{\sim}$ exists for $\mu$-a.e. $x \in C_{a, b}[0, T]$.

(2) The PWZ type stochastic integral $(w, x)_{C_{a, b}^{\prime}}^{\sim}$ is essentially independent of the choice of the complete orthonormal set $\left\{g_{j}\right\}_{j=1}^{\infty}$ in $C_{a, b}^{\prime}[0, T]$.

(3) It follows from the definition of the PWZ type stochastic integral and from Parseval's equality that if $w \in C_{a, b}^{\prime}[0, T]$ and $x \in C_{a, b}^{\prime}[0, T]$, then $(w, x)_{C_{a, b}^{\prime}}^{\sim}$ exists and we have $(w, x)_{C_{a, b}^{\prime}}^{\tilde{y}^{\prime}}=(w, x)_{C_{a, b}^{\prime}}$.

(4) If $D w=z \in L_{a, b}[0, T]$ is of bounded variation on $[0, T]$, then the PWZ type stochastic integral $(w, x)_{C_{a, b}^{\prime}}^{\sim}$ equals the Riemann-Stieltjes integral $\int_{0}^{T} z(t) d x(t)$ for $\mu$-a.e. $x \in C_{a, b}[0, T]$.

(5) The PWZ type stochastic integral has the expected linearity properties. That is, for any real number $c$, $w \in C_{a, b}^{\prime}[0, T]$ and $x \in C_{a, b}[0, T]$, we have

$$
(w, c x)_{C_{a, b}^{\prime}}^{\sim}=c(w, x)_{C_{a, b}^{\prime}}^{\tilde{a}^{\prime}}=(c w, x)_{C_{a, b}^{\prime}}^{\sim} .
$$

Thus, the assertions (1) and (4) hold for s-a.e. $x \in$ $C_{a, b}[0, T]$.

(6) For each $w \in C_{a, b}^{\prime}[0, T],(w, x)_{\tilde{C}_{a b}^{\prime}}$ is a Gaussian random variable with mean $(w, a)_{C_{a, b}^{\prime}}$ and variance $\|w\|_{C_{a, b}^{\prime}}^{2}$. For all $w_{1}, w_{2} \in C_{a, b}^{\prime}[0, T]$, we have

$$
\begin{aligned}
& \int_{C_{a, b}[0, T]}\left(w_{1}, x\right)_{C_{a, b}^{\prime}}^{\sim}\left(w_{2}, x\right)_{C_{a, b}^{\prime}}^{\sim} d \mu(x) \\
& =\left(w_{1}, w_{2}\right)_{C_{a, b}^{\prime}}+\left(w_{1}, a\right)_{C_{a, b}^{\prime}}\left(w_{2}, a\right)_{C_{a, b}^{\prime}} .
\end{aligned}
$$

Now, we are ready to examine a representation for paths $x$ in $C_{a, b}[0, T]$. Throughout the rest of this paper, we will use the symbol $\mathscr{G}$ for a complete orthonormal set $\left\{g_{j}\right\}_{j=1}^{\infty}$ in $C_{a, b}^{\prime}[0, T]$, such that for each $j=1,2, \ldots, D g_{j}=\alpha_{j}$ is of bounded variation on $[0, T]$. Then, for each $j=1,2, \ldots$,

$$
\gamma_{j}(x) \equiv\left(g_{j}, x\right)_{C_{a, b}^{\prime}}^{\sim}=\int_{0}^{T} \alpha_{j}(t) d x(t)
$$

is a Gaussian random variable with mean

$$
A_{j} \equiv\left(g_{j}, a\right)_{C_{a, b}^{\prime}}=\int_{0}^{T} \alpha_{j}(t) d a(t)
$$

and variance

$$
\left\|g_{j}\right\|_{C_{a, b}^{\prime}}^{2}=\int_{0}^{T} \alpha_{j}^{2}(t) d b(t)=1 .
$$

We note that the set $\left\{\gamma_{1}(x), \gamma_{2}(x), \ldots\right\}$ forms a set of independent Gaussian random variables on $C_{a, b}[0, T]$.

Let $\mathscr{G} \equiv\left\{g_{j}\right\}_{j=1}^{\infty}$ be as above, and let $x \in C_{a, b}[0, T]$. For each $n=1,2, \ldots$, define

$$
Z_{n}(x)(t) \equiv \sum_{j=1}^{n} \gamma_{j}(x) g_{j}(t) .
$$

Then, $Z_{n}(x)$ is an element of $C_{a, b}^{\prime}[0, T]$ for all $x \in C_{a, b}[0, T]$ and all $n=1,2, \ldots$.

For each $t \in[0, T]$, let $\Psi_{t}(s)=\int_{0}^{s} \chi_{[0, t]}(\tau) d b(\tau)$. Then, we observe that for each $t \in[0, T]$,

$$
\begin{aligned}
x(t) & =\int_{0}^{T} \chi_{[0, t]}(s) d x(s) \\
& =\left(\Psi_{t}, x\right)_{C_{a, b}^{\prime}}^{\sim} \\
& =\lim _{n \rightarrow \infty} \sum_{j=1}^{n}\left(\Psi_{t}, g_{j}\right)_{C_{a, b}^{\prime}} \int_{0}^{T} \alpha_{j}(s) d x(s) \\
& =\lim _{n \rightarrow \infty} \sum_{j=1}^{n} \int_{0}^{t} \alpha_{j}(s) d b(s) \gamma_{j}(x) \\
& =\lim _{n \rightarrow \infty} \sum_{j=1}^{n} \gamma_{j}(x) g_{j}(t) \\
& =\lim _{n \rightarrow \infty} Z_{n}(x)(t) .
\end{aligned}
$$

By the property of the PWZ type stochastic integral, the last expression of (20) converges for $\mu$-a.e. $x \in C_{a, b}[0, T]$.

Remark 2. By the definition of the PWZ type stochastic integral, the last expression of (20) is independent of the choice of the complete orthonormal set $\mathscr{G}$ in $C_{a, b}^{\prime}[0, T]$. If we choose the complete orthonormal sine sequence $\mathscr{G} \equiv\left\{g_{j}\right\}_{j=1}^{\infty}$ in $C_{a, b}^{\prime}[0, T]$, where

$$
g_{j}(t)=\frac{\sqrt{2 b(T)}}{(j-1 / 2) \pi} \sin \left[\frac{(j-1 / 2) \pi b(t)}{b(T)}\right] ;
$$


that is,

$$
\alpha_{j}(t)=D g_{j}(t)=\sqrt{\frac{2}{b(T)}} \cos \left[\frac{(j-1 / 2) \pi b(t)}{b(T)}\right]
$$

for $j=1,2, \ldots$, then the corresponding $Z_{n}(x)(t)$ converges to $x(t)$ uniformly in $t$ with probability one. For more details, see [20] and the references therein.

We now state a fundamental integration formula on the function space $C_{a, b}[0, T]$. Let $\gamma_{j}(x)$ and $A_{j}$ be as above, let $h: \mathbb{R}^{n} \rightarrow \mathbb{C}$ be Lebesgue measurable, and let $H(x)=$ $h\left(\gamma_{1}(x), \ldots, \gamma_{n}(x)\right)$. Then

$$
\begin{aligned}
& \int_{C_{a, b}[0, T]} H(x) d \mu(x) \\
& \equiv \int_{C_{a, b}[0, T]} h\left(\gamma_{1}(x), \ldots, \gamma_{n}(x)\right) d \mu(x) \\
& =(2 \pi)^{-n / 2} \int_{\mathbb{R}^{n}} h\left(u_{1}, \ldots, u_{n}\right) \\
& \quad \times \exp \left\{-\sum_{j=1}^{n} \frac{\left[u_{j}-A_{j}\right]^{2}}{2}\right\} d u_{1} \ldots d u_{n}
\end{aligned}
$$

in the sense that if either side of (23) exists, both sides exist and equality holds.

Let $F$ be a functional on $C_{a, b}[0, T]$ and let $\mathscr{G} \equiv\left\{g_{j}\right\}_{j=1}^{\infty}$ be a complete orthonormal set in $C_{a, b}^{\prime}[0, T]$. Then, we say that $F$ is $\mathscr{G}$-continuous at $x$ if

$$
\lim _{n \rightarrow \infty} F\left(Z_{n}(x)\right)=F(x)
$$

where $Z_{n}(x)$ is given by (19).

Example 3. For each $t \in[0, T]$, let $F: C_{a, b}[0, T] \rightarrow \mathbb{R}$ be given by $F(x)=x(t)$. Then, using (20) and (14), we see that $F$ is $\mathscr{G}$-continuous for s-a.e. $x \in C_{a, b}[0, T]$ and all $x \in C_{a, b}^{\prime}[0, T]$.

Example 4. Given $w=D^{-1} z \in C_{a, b}^{\prime}[0, T]$, let $F$ : $C_{a, b}[0, T] \rightarrow \mathbb{R}$ be given by $F(x)=(w, x)_{C_{a, b}^{\prime}}^{\sim}$. Since $(w, x)_{C_{a, b}^{\prime}}^{\tilde{y}^{\prime}}=(w, x)_{C_{a, b}^{\prime}}$ for every $x \in C_{a, b}^{\prime}[0, T]$, by the definition of PWZ type stochastic integral, we obtain that

$$
\begin{aligned}
\lim _{n \rightarrow \infty} F\left(Z_{n}(x)\right) & =\lim _{n \rightarrow \infty}\left(w, Z_{n}(x)\right)_{C_{a, b}^{\prime}} \\
& =\lim _{n \rightarrow \infty} \int_{0}^{T} z(t) d Z_{n}(x)(t) \\
& =\lim _{n \rightarrow \infty} \int_{0}^{T} z(t) d\left(\sum_{j=1}^{n} \gamma_{j}(x) g_{j}(t)\right)
\end{aligned}
$$

$$
\begin{aligned}
& =\lim _{n \rightarrow \infty} \sum_{j=1}^{n} \gamma_{j}(x) \int_{0}^{T} z(t) d g_{j}(t) \\
& =\lim _{n \rightarrow \infty} \sum_{j=1}^{n}\left(z, \alpha_{j}\right)_{0, b} \gamma_{j}(x) \\
& =(w, x)_{C_{a, b}^{\prime}}^{\sim}=F(x)
\end{aligned}
$$

for s-a.e. $x \in C_{a, b}[0, T]$ and all $x \in C_{a, b}^{\prime}[0, T]$.

Proposition 5. Let $F: C_{a, b}[0, T] \rightarrow \mathbb{C}$ be $\mu$-integrable. Assume that $F$ is $\mathscr{G}$-continuous for $\mu$-a.e. $x \in C_{a, b}[0, T]$. Then

$$
\begin{aligned}
& \int_{C_{a, b}[0, T]} F(x) d \mu(x) \\
& \quad=\lim _{n \rightarrow \infty} \int_{C_{a, b}[0, T]} F\left(Z_{n}(x)\right) d \mu(x),
\end{aligned}
$$

where $Z_{n}(x)$ is given by (19).

Proof. For each $n=1,2, \ldots$, let $F_{n}(x)=F\left(Z_{n}(x)\right)$. Then $F_{n}$ is $\mu$-integrable. By our assumption, we observe that

$$
\lim _{n \rightarrow \infty} F_{n}(x)=\lim _{n \rightarrow \infty} F\left(Z_{n}(x)\right)=F(x)
$$

for $\mu$-a.e. $x \in C_{a, b}[0, T]$. Thus, by the dominated convergence theorem, it follows that

$$
\begin{aligned}
& \lim _{n \rightarrow \infty} \int_{C_{a, b}[0, T]} F\left(Z_{n}(x)\right) d \mu(x) \\
& =\lim _{n \rightarrow \infty} \int_{C_{a, b}[0, T]} F_{n}(x) d \mu(x) \\
& =\int_{C_{a, b}[0, T]} \lim _{n \rightarrow \infty} F_{n}(x) d \mu(x) \\
& =\int_{C_{a, b}[0, T]} F(x) d \mu(x)
\end{aligned}
$$

as desired.

\section{Sequential Function Space Integrals}

In [21], Cameron and Storvick defined the "sequential" Feynman integral by means of finite dimensional approximations for functionals on Wiener space $C_{0}[0, T]$. The sequential definition for the Feynman integral was intended to interpret the Feynman's uniform measure [22] on path space $C_{0}[0, T]$, because there is no countably additive measure which weights all paths $x$ in $C_{0}[0, T]$ equally in much the same way as Lebesgue measure weights all points in $\mathbb{R}$ equally. Thus, the Cameron and Storvick's sequential Feynman integral is a rigorous mathematical formulation of the Feynman's path integral.

The Cameron and Storvick's sequential Feynman integral is related by sequential Wiener integral [23]; that is, the 
integral is based on polygonal path approximations. In this section, we define different kinds of sequential function space integrals for functionals on the function space $C_{a, b}[0, T]$. In Section 4 below, we also adopt sequential approaches to define our function space transforms on $C_{a, b}[0, T]$. The sequential definition for the Feynman integral in [21] was defined as the limit of a sequence of finite dimensional Lebesgue integrals. Essentially, our sequential function space integrals and transforms are defined in terms of a sequence of complex measures on the function space $C_{a, b}[0, T]$.

Next, we introduce two sequential definitions for certain function space integrals on $C_{a, b}[0, T]$. Throughout the rest of this paper, let $\mathbb{C}_{+}$and $\widetilde{\mathbb{C}}_{+}$denote the set of complex numbers with positive real part and nonzero complex numbers with nonnegative real part, respectively. Furthermore, for all $\lambda \epsilon$ $\mathbb{C}, \lambda^{1 / 2}$ is always chosen to have nonnegative real part.

Let $\mathscr{G} \equiv\left\{g_{j}\right\}_{j=1}^{\infty}, \gamma_{j}(x)$, and $A_{j}$ be as in Section 2. For $\lambda \in$ $\mathbb{C}_{+}, x \in C_{a, b}[0, T]$ and each $n=1,2, \ldots$, let

$$
\begin{aligned}
& W_{n}^{+}(\lambda ; x) \equiv \lambda^{n / 2} \exp \left\{\left(\frac{1-\lambda}{2}\right) \sum_{j=1}^{n} \gamma_{j}^{2}(x)\right. \\
&\left.+\left(\lambda^{1 / 2}-1\right) \sum_{j=1}^{n} A_{j} \gamma_{j}(x)\right\}
\end{aligned}
$$

and let

$$
\begin{aligned}
W_{n}^{-}(\lambda ; x) \equiv \lambda^{n / 2} \exp & \left\{\left(\frac{1-\lambda}{2}\right) \sum_{j=1}^{n} \gamma_{j}^{2}(x)\right. \\
& \left.+\left((-\lambda)^{1 / 2}-1\right) \sum_{j=1}^{n} A_{j} \gamma_{j}(x)+\sum_{j=1}^{n} A_{j}^{2}\right\} .
\end{aligned}
$$

Using (23) and (11), we observe that for all $\lambda \in \mathbb{C}_{+}$and every $n=1,2, \ldots$,

$$
\begin{aligned}
& \int_{C_{a, b}[0, T]} W_{n}^{+}(\lambda ; x) d \mu(x) \\
& \quad=\int_{C_{a, b}[0, T]} W_{n}^{-}(\lambda ; x) d \mu(x)=1 .
\end{aligned}
$$

We are now ready to state the definition of the sequential function space integrals.

Definition 6. Let $F$ be a measurable functional on $C_{a, b}[0, T]$. Let $q \neq 0$ be a real number and let $\left\{\lambda_{n}\right\}_{n=1}^{\infty}$ be a sequence of complex numbers in $\mathbb{C}_{+}$such that $\lambda_{n} \rightarrow-i q$. If the following limit exists, one calls it the sequential $\mathscr{P}$-function space integral of $F$ with parameter $q$, and we write

$$
\begin{aligned}
& \int_{C_{a, b}[0, T]}^{P_{q}} F(x) d^{\text {se }} x \\
& \quad=\lim _{n \rightarrow \infty} \int_{C_{a, b}[0, T]} F\left(Z_{n}(x)\right) W_{n}^{+}\left(\lambda_{n} ; x\right) d \mu(x) .
\end{aligned}
$$

We also define the sequential $\mathcal{N}$-function space integral of $F$ with parameter $q$ by the formula

$$
\begin{aligned}
& \int_{C_{a, b}[0, T]}^{N_{q}} F(x) d^{\mathrm{se}} x \\
& \quad=\lim _{n \rightarrow \infty} \int_{C_{a, b}[0, T]} F\left(Z_{n}(x)\right) W_{n}^{-}\left(\lambda_{n} ; x\right) d \mu(x)
\end{aligned}
$$

if it exists.

$$
\begin{aligned}
& \text { Let } F_{1}(x) \equiv 1 \text { on } C_{a, b}[0, T] \text { and let } \\
& \qquad F_{2}(x)= \begin{cases}0, & x \in C_{a, b}^{\prime}[0, T], \\
1, & x \in C_{a, b}[0, T]-C_{a, b}^{\prime}[0, T] .\end{cases}
\end{aligned}
$$

Then, since $C_{a, b}^{\prime}[0, T]$ is a scale invariant null set, we have $F_{1} \approx F_{2}$. But by the definition of the sequential function space integrals, we see that

$$
\begin{aligned}
& \int_{C_{a, b}[0, T]}^{P_{q}} F_{1}(x) d^{\mathrm{se}} x=\int_{C_{a, b}[0, T]}^{N_{q}} F_{1}(x) d^{\mathrm{se}} x=1, \\
& \int_{C_{a, b}[0, T]}^{P_{q}} F_{2}(x) d^{\mathrm{se}} x=\int_{C_{a, b}[0, T]}^{N_{q}} F_{2}(x) d^{\mathrm{se}} x=0 .
\end{aligned}
$$

Given two complex-valued measurable functionals $F$ and $G$ on $C_{a, b}[0, T]$, we will write $F \cong G$ if $F \approx G$ and furthermore if $F(x)=G(x)$ for all $x \in C_{a, b}^{\prime}[0, T]$. The relation $\approx$ is clearly an equivalence relation.

Definition 7. Let $F$ be a functional on $C_{a, b}[0, T]$. If $F$ is $\mathscr{G}$ continuous for s-a.e. $x \in C_{a, b}[0, T]$ and every $x \in C_{a, b}^{\prime}[0, T]$, then one says that $F$ is $\mathscr{G}$-s-continuous.

The functionals discussed in Examples 3 and 4 above are $\mathscr{G}$-s-continuous. Next we introduce a class of functionals which are $\mathscr{G}$-s-continuous.

Let $\mathscr{M}\left(C_{a, b}^{\prime}[0, T]\right)$ be the space of complex-valued, countably additive (and hence finite) Borel measures on $C_{a, b}^{\prime}[0, T]$. $\mathscr{M}\left(C_{a, b}^{\prime}[0, T]\right)$ is a Banach algebra under the total variation norm and with convolution as multiplication.

Definition 8 . The Fresnel type class $\mathscr{F}^{*}\left(C_{a, b}[0, T]\right)$ is the space of functionals on $C_{a, b}[0, T]$ expressible in the form

$$
F(x) \cong \int_{C_{a, b}^{\prime}[0, T]} \exp \left\{i(w, x)_{C_{a, b}^{\prime}}^{\sim}\right\} d f(w)
$$

for s-a.e. $x \in C_{a, b}[0, T]$ and every $x \in C_{a, b}^{\prime}[0, T]$, where the associated measure $f$ is an element of $\mathscr{M}\left(C_{a, b}^{\prime}[0, T]\right)$. More precisely, since we will identify functionals which coincide under the relation $\approx$ on $C_{a, b}[0, T], \mathscr{F}^{*}\left(C_{a, b}[0, T]\right)$ can be regarded as the space of all $\cong$-equivalence classes of functionals of the form (36).

The Fresnel type class $\mathscr{F}^{*}\left(C_{a, b}[0, T]\right)$ is a Banach algebra with norm

$$
\|F\|=\|f\|=\int_{C_{a, b}^{\prime}[0, T]} d|f|(w) .
$$


In fact, the correspondence $f \mapsto F$ is injective, carries convolution into pointwise multiplication, and is a Banach algebra isomorphism where $f$ and $F$ are related by (36).

Proposition 9. Let $F \in \mathscr{F}^{*}\left(C_{a, b}[0, T]\right)$. Then $F$ is $\mathscr{G}-s-$ continuous.

Proof. Let $F$ be given by (36), and for each $n=1,2, \ldots$, let $Z_{n}(x)$ be given by (19). Then substituting $Z_{n}(x)$ for $x$, we have

$$
F\left(Z_{n}(x)\right)=\int_{C_{a, b}^{\prime}[0, T]} \exp \left\{i\left(w, Z_{n}(x)\right)_{C_{a, b}^{\prime}}^{\sim}\right\} d f(w) .
$$

By Example 4, the exponential in (38) approaches the exponential in equation (36) as $n \rightarrow \infty$. Note that for each $n \in \mathbb{N}$, the exponential in (38) is measurable in $(w, x)$ on $C_{a, b}^{\prime}[0, T] \times$ $C_{a, b}[0, T]$. Thus, by the bounded convergence theorem, we observe $F\left(Z_{n}(x)\right) \rightarrow F(x)$ for s-a.e $x \in C_{a, b}[0, T]$ and every $x \in C_{a, b}^{\prime}[0, T]$, and the proposition is proved.

The functional $F_{2}$ defined by equation (34) above is not $\mathscr{G}$-s-continuous. Thus, $F_{2} \notin \mathscr{F}^{*}\left(C_{a, b}^{\prime}[0, T]\right)$.

The following lemma plays a key role in the proofs of Propositions 12 and 13 below.

Lemma 10. For each $n=1,2, \ldots$, let $Z_{n}(x)$ be given by (19) and let $W_{n}^{+}$and $W_{n}^{-}$be given by (29) and (30), respectively. Then for all $w \in C_{a, b}^{\prime}[0, T]$, all $\lambda \in \mathbb{C}_{+}$, and each $n=1,2, \ldots$, the function space integrals

$$
\begin{aligned}
& \int_{C_{a, b}[0, T]} \exp \left\{i\left(w, Z_{n}(x)\right)_{C_{a, b}^{\prime}}^{\sim}\right\} W_{n}^{+}(\lambda ; x) d \mu(x), \\
& \int_{C_{a, b}[0, T]} \exp \left\{i\left(w, Z_{n}(x)\right)_{C_{a, b}^{\prime}}^{\sim}\right\} W_{n}^{-}(\lambda ; x) d \mu(x)
\end{aligned}
$$

exist and are given by (40) and (41) below, respectively.

Proof. Using (19), (29), (23), the Fubini theorem, and (11), we obtain

$$
\begin{aligned}
\int_{C_{a, b}[0, T]} \exp \left\{i\left(w, Z_{n}(x)\right)_{C_{a, b}^{\prime}}^{\sim}\right\} W_{n}^{+}(\lambda ; x) d \mu(x) \\
=\left(\frac{\lambda}{2 \pi}\right)^{n / 2} \int_{\mathbb{R}^{n}} \exp \left\{-\frac{\lambda}{2} \sum_{j=1}^{n} u_{j}^{2}\right. \\
+\sum_{j=1}^{n}\left(i\left(w, g_{j}\right)_{C_{a, b}^{\prime}}+\lambda^{1 / 2} A_{j}\right) u_{j} \\
\left.-\frac{1}{2} \sum_{j=1}^{n} A_{j}^{2}\right\} d u_{1} \ldots d u_{n}
\end{aligned}
$$

$$
\begin{aligned}
=\exp \{ & -\frac{1}{2 \lambda} \sum_{j=1}^{n}\left(w, g_{j}\right)_{C_{a, b}^{\prime}}^{2} \\
& \left.+i \lambda^{-1 / 2} \sum_{j=1}^{n}\left(w, g_{j}\right)_{C_{a, b}^{\prime}} A_{j}\right\} .
\end{aligned}
$$

Also, using (19), (30), (23), the Fubini theorem, and (11), we obtain

$$
\begin{gathered}
\int_{C_{a, b}[0, T]} \exp \left\{i\left(w, Z_{n}(x)\right)_{\widetilde{C}_{a, b}^{\prime}}\right\} W_{n}^{-}(\lambda ; x) d \mu(x) \\
=\exp \left\{-\frac{1}{2 \lambda} \sum_{j=1}^{n}\left(w, g_{j}\right)_{C_{a, b}^{\prime}}^{2}\right. \\
\left.-i(-\lambda)^{-1 / 2} \sum_{j=1}^{n}\left(w, g_{j}\right)_{C_{a, b}^{\prime}} A_{j}\right\} .
\end{gathered}
$$

Let $F$ be given by (36). Proceeding formally using (36), (40), and (41), we see that the sequential function space integrals $\int_{C_{a, b}[0, T]}^{P_{q}} F(x) d^{\mathrm{se}} x$ and $\int_{C_{a, b}[0, T]}^{N_{q}} F(x) d^{\mathrm{se}} x$ are given by the formulas

$$
\begin{aligned}
& \int_{C_{a, b}[0, T]}^{P_{q}} F(x) d^{\mathrm{se} x} \\
& =\int_{C_{a, b}^{\prime}[0, T]} \exp \left\{-\frac{i}{2 q}\|w\|_{C_{a, b}^{\prime}}^{2}\right. \\
& \left.+i(-i q)^{-1 / 2}(w, a)_{C_{a, b}^{\prime}}\right\} d f(w), \\
& \int_{C_{a, b}[0, T]}^{N_{q}} F(x) d^{\mathrm{se}} x \\
& =\int_{C_{a, b}^{\prime}[0, T]} \exp \left\{-\frac{i}{2 q}\|w\|_{C_{a, b}^{\prime}}^{2}\right. \\
& \left.-i(i q)^{-1 / 2}(w, a)_{C_{a, b}^{\prime}}\right\} d f(w),
\end{aligned}
$$

respectively.

$$
\begin{aligned}
& \text { For } \lambda \in \widetilde{\mathbb{C}}_{+} \text {and } w \in C_{a, b}^{\prime}[0, T] \text {, let } \\
& \psi_{P}(\lambda ; w) \equiv \exp \left\{-\frac{1}{2 \lambda}\|w\|_{C_{a, b}^{\prime}}^{2}+i \lambda^{-1 / 2}(w, a)_{C_{a, b}^{\prime}}\right\} \\
& \psi_{N}(\lambda ; w) \equiv \exp \left\{-\frac{1}{2 \lambda}\|w\|_{C_{a, b}^{\prime}}^{2}-i(-\lambda)^{-1 / 2}(w, a)_{C_{a, b}^{\prime}}\right\} .
\end{aligned}
$$

Then, for each $\lambda \in \widetilde{\mathbb{C}}_{+},\left|\psi_{P}(\lambda ; w)\right|$ and $\left|\psi_{N}(\lambda ; w)\right|$ are unbounded functions of $w$. Hence, $\int_{C_{a, b}[0, T]}^{P_{q}} F(x) d^{\text {se }} x$ and $\int_{C_{a, b}[0, T]}^{N_{q}} F(x) d^{\mathrm{se}} x$ might not exist. From this observation, we 
clearly need to impose additional restrictions, such as (45) below, on the functionals $F$ in $\mathscr{F}^{*}\left(C_{a, b}[0, T]\right)$ for the existence of the sequential $\mathscr{P}$ and $\mathscr{N}$-function space integrals of $F$.

Let $q_{0}$ be a positive real number. We define a subclass $\mathscr{F}_{q_{0}}^{*} \equiv \mathscr{F}_{q_{0}}^{*}\left(C_{a, b}[0, T]\right)$ of $\mathscr{F}^{*}\left(C_{a, b}[0, T]\right)$ by $F \in \mathscr{F}_{q_{0}}^{*}$ if and only if

$$
\int_{C_{a, b}^{\prime}[0, T]} \exp \left\{\frac{1}{\sqrt{2 q_{0}}}\|w\|_{C_{a, b}^{\prime}}\|a\|_{C_{a, b}^{\prime}}\right\} d|f|(w)<+\infty
$$

where $f$ and $F$ are related by (36).

The following example suggests the necessity of the condition (45) to ensure the existence of the sequential function space integrals of functionals in $\mathscr{F}^{*}\left(C_{a, b}[0, T]\right)$.

For each $n \in \mathbb{N}$, let $w_{n}(t)=n b(t)=\int_{0}^{t} n d b(s)$ for $t \in[0, T]$. Consider a measure $\eta$ on $C_{a, b}^{\prime}[0, T]$ which is concentrated on the set $\left\{w_{n}: n \in \mathbb{N}\right\}$ and $\eta\left(\left\{w_{n}\right\}\right)=1 /\left(n^{2}\right)$ for each $n \in \mathbb{N}$. Then, $\eta$ is an element of $\mathscr{M}\left(C_{a, b}^{\prime}[0, T]\right)$ and it follows that

$$
\begin{aligned}
& \int_{C_{a, b}^{\prime}[0, T]} \exp \left\{\frac{1}{\sqrt{2 q_{0}}}\|w\|_{C_{a, b}^{\prime}}\|a\|_{C_{a, b}^{\prime}}\right\} d|\eta|(w) \\
& =\sum_{n=1}^{\infty} \exp \left\{\frac{1}{\sqrt{2 q_{0}}}\left\|w_{n}\right\|_{C_{a, b}^{\prime}}\|a\|_{C_{a, b}^{\prime}}\right\} \frac{1}{n^{2}} \\
& =\sum_{n=1}^{\infty} \exp \left\{\frac{\sqrt{b(T)}\|a\|_{C_{a, b}^{\prime}}}{\sqrt{2 q_{0}}}\right\} \frac{1}{n^{2}} \\
& \geq \sum_{n=1}^{\infty} \frac{\sqrt{b(T)}\|a\|_{C_{a, b}^{\prime}}}{\frac{\sqrt{2 q_{0}}}{n}}=+\infty .
\end{aligned}
$$

Using the same method, we can find an example for the functional in $\mathscr{F}^{*}\left(C_{a, b}[0, T]\right)$ that the sequential integrals do not exist.

Given a positive real number $q_{0}$, let

$$
\Gamma_{q_{0}}=\left\{\lambda \in \widetilde{\mathbb{C}}_{+}|\lambda=\alpha+i \beta,| \operatorname{Im}\left(\lambda^{-1 / 2}\right) \mid<\frac{1}{\sqrt{2 q_{0}}}\right\} .
$$

Let $q$ be a real number with $|q|>q_{0}$. Then, since

$$
\begin{aligned}
& (-i q)^{-1 / 2}=\frac{1}{\sqrt{2|q|}}+i \frac{\operatorname{sign}(q)}{\sqrt{2|q|}}, \\
& (i q)^{-1 / 2}=\frac{1}{\sqrt{2|q|}}-i \frac{\operatorname{sign}(q)}{\sqrt{2|q|}},
\end{aligned}
$$

we can see that $-i q$ and $i q$ are elements of the domain $\operatorname{Int}\left(\Gamma_{q_{0}}\right)$.

We also need the following lemma to obtain Propositions 12 and 13 .

Lemma 11. Let $\mathscr{G} \equiv\left\{g_{j}\right\}_{j=1}^{\infty}$ and $A_{j}$ be as in Section 2, and let $q_{0}$ be a positive real number. Let $\left\{\lambda_{n}\right\}_{n=1}^{\infty}$ be a sequence in $\mathbb{C}_{+}$ such that $\lambda \rightarrow-i q$, where $q$ is a real number with $|q|>q_{0}$.
Then there exists a sufficiently large $K \in \mathbb{N}$ such that for all $n \geq K$,

$$
\begin{aligned}
& \exp \left\{\left|\operatorname{Im}\left(\lambda_{n}^{-1 / 2}\right)\right|\left|\sum_{j=1}^{n}\left(g_{j}, w\right)_{C_{a, b}^{\prime}} A_{j}\right|\right\} \\
& <\exp \left\{\frac{1}{\sqrt{2 q_{0}}}\|w\|_{C_{a, b}^{\prime}}\|a\|_{C_{a, b}^{\prime}}\right\}, \\
& \exp \left\{\left|\operatorname{Im}\left(\left(-\lambda_{n}\right)^{-1 / 2}\right)\right|\left|\sum_{j=1}^{n}\left(g_{j}, w\right)_{C_{a, b}^{\prime}} A_{j}\right|\right\} \\
& \quad<\exp \left\{\frac{1}{\sqrt{2 q_{0}}}\|w\|_{C_{a, b}^{\prime}}\|a\|_{C_{a, b}^{\prime}}\right\} .
\end{aligned}
$$

Proof. Let $\left\{\lambda_{n}\right\}_{n=1}^{\infty}=\left\{\alpha_{n}+i \beta_{n}\right\}_{n=1}^{\infty}$ be a sequence in $\mathbb{C}_{+}$such that $\lambda_{n} \rightarrow-i q$, and let $\Gamma_{q_{0}}$ be given by (47). Then, we observe that for each $n \in \mathbb{N}$,

$$
\begin{aligned}
& \operatorname{Im}\left(\lambda_{n}^{-1 / 2}\right)=-\operatorname{sign}\left(\beta_{n}\right) \sqrt{\frac{\sqrt{\alpha_{n}^{2}+\beta_{n}^{2}}-\alpha_{n}}{2\left(\alpha_{n}^{2}+\beta_{n}^{2}\right)}}, \\
& \operatorname{Im}\left(\left(-\lambda_{n}\right)^{-1 / 2}\right)=\operatorname{sign}\left(\beta_{n}\right) \sqrt{\frac{\sqrt{\alpha_{n}^{2}+\beta_{n}^{2}}+\alpha_{n}}{2\left(\alpha_{n}^{2}+\beta_{n}^{2}\right)}} .
\end{aligned}
$$

Since $\alpha_{n} \rightarrow 0$, there exists a sufficiently large $K_{1} \in \mathbb{N}$ such that for every $n \geq K_{1}, \lambda_{n} \in \operatorname{Int}\left(\Gamma_{q_{0}}\right)$ and

$$
\begin{aligned}
& \delta(q)=\sup (\left\{\left|\operatorname{Im}\left(\lambda_{n}^{-1 / 2}\right)\right|: n \geq K_{1}\right\} \\
&\left.\cup\left\{\left|\operatorname{Im}\left(\left(-\lambda_{n}\right)^{-1 / 2}\right)\right|: n \geq K_{1}\right\} \cup\left\{\frac{1}{\sqrt{2|q|}}\right\}\right) \\
&<\frac{1}{\sqrt{2 q_{0}}} .
\end{aligned}
$$

Also, there exists a positive real number $\varepsilon_{0}>1$ such that $\delta(q)<1 /\left(\varepsilon_{0} \sqrt{2 q_{0}}\right)$.

Let $\mathscr{G} \equiv\left\{g_{j}\right\}_{j=1}^{\infty}$ and $A_{j}$ be as in Section 2 above. Using Parseval's identity, we observe

$$
\begin{aligned}
(w, a)_{C_{a, b}^{\prime}} & =\sum_{j=1}^{\infty}\left(w, g_{j}\right)_{C_{a, b}^{\prime}}\left(g_{j}, a\right)_{C_{a, b}^{\prime}} \\
& =\sum_{j=1}^{\infty}\left(w, g_{j}\right)_{C_{a, b}^{\prime}} A_{j}
\end{aligned}
$$

for $w \in C_{a, b}^{\prime}[0, T]$. Also, using the Cauchy-Schwartz inequality, we have

$$
-\varepsilon_{0}\|w\|_{C_{a, b}^{\prime}}\|a\|_{C_{a, b}^{\prime}}<(w, a)_{C_{a, b}^{\prime}}<\varepsilon_{0}\|w\|_{C_{a, b}^{\prime}}\|a\|_{C_{a, b}^{\prime}} .
$$


Thus, there exists a sufficiently large $K_{2} \in \mathbb{N}$ such that for every $n \geq K_{2}$,

$$
\left|\sum_{j=1}^{n}\left(g_{j}, w\right)_{C_{a, b}^{\prime}} A_{j}\right|<\varepsilon_{0}\|w\|_{C_{a, b}^{\prime}\|a\|_{C_{a, b}^{\prime}}} .
$$

Using these facts, we obtain the inequality (49) and (50).

Proposition 12. Let $q_{0}$ be a positive real number and let $F \in$ $\mathscr{F}_{q_{0}}^{*}$ be given by (36). Then for all real $q$ with $|q|>q_{0}$, the sequential $\mathscr{P}$-function space integral $\int_{C_{a, b}[0, T]}^{P_{q}} F(x) d^{\text {se }} x$ exists and is given by the formula

$$
\int_{C_{a, b}[0, T]}^{P_{q}} F(x) d^{\text {se }} x=\int_{C_{a, b}^{\prime}[0, T]} \psi_{P}(-i q ; w) d f(w),
$$

where $\psi_{P}$ is given by (43) above.

Proof. Let $F$ be given by (36). First note that the equality in (36) holds for all $x \in C_{a, b}^{\prime}[0, T]$. Let $q$ be a real number with $|q|>q_{0}$, and let $\left\{\lambda_{n}\right\}_{n=1}^{\infty}$ be a sequence in $\mathbb{C}_{+}$which converges to -iq. Using (36), (19), (29), and the Fubini theorem, we obtain that for each $n=1,2, \ldots$,

$$
\begin{aligned}
& \int_{C_{a, b}[0, T]} F\left(Z_{n}(x)\right) W_{n}^{+}\left(\lambda_{n} ; x\right) d \mu(x) \\
&=\int_{C_{a, b}^{\prime}[0, T]} \int_{C_{a, b}[0, T]} \exp \left\{i\left(w, Z_{n}(x){\tilde{C_{a, b}^{\prime}}}^{\sim}\right\}\right. \\
& \times W_{n}^{+}\left(\lambda_{n} ; x\right) d \mu(x) d f(w) .
\end{aligned}
$$

But, by (40) and (49), we know that the right hand side of (57) is dominated by (45) for all but a finite number of values of $n$. Next, using (57), (40) with $\lambda$ replaced with $\lambda_{n}$, the dominated convergence theorem, Parseval's relation, and (43), we obtain

$$
\begin{gathered}
\lim _{n \rightarrow \infty} \int_{C_{a, b}[0, T]} F\left(Z_{n}(x)\right) W_{n}^{+}\left(\lambda_{n} ; x\right) d \mu(x) \\
=\int_{C_{a, b}^{\prime}[0, T]} \lim _{n \rightarrow \infty} \exp \left\{-\frac{1}{2 \lambda_{n}} \sum_{j=1}^{n}\left(w, g_{j}\right)_{C_{a, b}^{\prime}}^{2}\right. \\
\left.+i \lambda_{n}^{-1 / 2} \sum_{j=1}^{n}\left(w, g_{j}\right)_{C_{a, b}^{\prime}} A_{j}\right\} d f(w) \\
=\int_{C_{a, b}^{\prime}[0, T]} \exp \left\{-\frac{i}{2 q}\|w\|_{C_{a, b}^{\prime}}^{2}\right. \\
\left.+i(-i q)^{-1 / 2}(w, a)_{C_{a, b}^{\prime}}\right\} d f(w)
\end{gathered}
$$

which concludes the proof of Proposition 12.

We establish our next proposition after careful examination of the proof of Proposition 12, and by using (30), (41), (44), and (50) instead of (29), (40), (43), and (49), respectively.
Proposition 13. Let $q_{0}$ and $F \in \mathscr{F}_{q_{0}}^{*}$ be as in Proposition 12. Then for all real $q$ with $|q|>q_{0}$, the sequential $\mathcal{N}$-function space integral $\int_{C_{a, b}[0, T]}^{N_{q}} F(x) d^{s e} x$ exists and is given by the formula

$$
\int_{C_{a, b}[0, T]}^{N_{q}} F(x) d^{s e} x=\int_{C_{a, b}^{\prime}[0, T]} \psi_{N}(-i q ; w) d f(w),
$$

where $\psi_{N}$ is given by (44).

\section{Sequential Function Space Transforms}

In this section, we introduce two sequential transforms on the function space $C_{a, b}[0, T]$. We then establish that each of these transforms acts like an inverse transform of the other transform. Our definitions of the sequential transforms are based on the sequential function space integrals defined in Section 3 above.

Definition 14. Let $q$ be a nonzero real number. For $y \in$ $C_{a, b}[0, T]$, we define the sequential $\mathscr{P}$-function space transform $\mathscr{P}_{q}(F)$ of $F$ with parameter $q$ by the formula

$$
\mathscr{P}_{q}(F)(y)=\int_{C_{a, b}[0, T]}^{P_{q}} F(y+x) d^{\text {se }} x
$$

if it exists. Also, we define the sequential $\mathcal{N}$-function space transform $\mathcal{N}_{q}(F)$ of $F$ with parameter $q$ by the formula

$$
\mathcal{N}_{q}(F)(y)=\int_{C_{a, b}[0, T]}^{N_{q}} F(y+x) d^{\text {se }} x
$$

if it exists.

In Theorem 15 below, we establish the existence of the sequential $\mathscr{P}$-function space transform of functionals in $\mathscr{F}^{*}\left(C_{a, b}[0, T]\right)$.

Theorem 15. Let $q_{0}$ and $F \in \mathscr{F}_{q_{0}}^{*}$ be as in Proposition 12. Then for all real $q$ with $|q|>q_{0}$, the sequential $\mathscr{P}$-function space transform of $F, \mathscr{P}_{q}(F)$ exists and is an element of $\mathscr{F}^{*}\left(C_{a, b}[0, T]\right)$ with associated measure $f_{q}^{P}$ defined by

$$
f_{q}^{P}(E)=\int_{E} \psi_{P}(-i q ; w) d f(w), \quad E \in \mathscr{B}\left(C_{a, b}^{\prime}[0, T]\right),
$$

where $\psi_{P}$ is given by (43), $\mathscr{B}\left(C_{a, b}^{\prime}[0, T]\right)$ is the Borel $\sigma$-algebra, and $f$ is the associated measure of $F$ by (36). Furthermore, one sees that for s-a.e. $y \in C_{a, b}[0, T]$ and all $y \in C_{a, b}^{\prime}[0, T]$,

$$
\begin{aligned}
& \mathscr{P}_{q}(F)(y) \\
& =\int_{C_{a, b}^{\prime}[0, T]} \exp \left\{i(w, y)_{C_{a, b}^{\prime}}^{\sim}\right\} \psi_{P}(-i q ; w) d f(w) \\
& =\int_{C_{a, b}^{\prime}[0, T]} \exp \left\{i(w, y)_{C_{a, b}^{\prime}}^{\sim}\right\} d f_{q}^{P}(w),
\end{aligned}
$$


with

$$
\begin{aligned}
\left\|f_{q}^{P}\right\| & <\int_{C_{a, b}^{\prime}[0, T]} \exp \left\{\frac{1}{\sqrt{2 q_{0}}}\|w\|_{C_{a, b}^{\prime}}\|a\|_{C_{a, b}^{\prime}}\right\} d|f|(w) \\
& <+\infty .
\end{aligned}
$$

Proof. Let $F$ be given by (36), and for a real $q$ with $|q|>$ $q_{0}$, let $\left\{\lambda_{n}\right\}_{n=1}^{\infty}$ be a sequence in $\mathbb{C}_{+}$which converges to $-i q$. Proceeding as in the proof of Proposition 12, we obtain that for s-a.e. $y \in C_{a, b}[0, T]$ and all $y \in C_{a, b}^{\prime}[0, T]$,

$$
\begin{aligned}
& \mathscr{P}_{q}(F)(y) \\
& =\lim _{n \rightarrow \infty} \int_{C_{a, b}[0, T]} F\left(y+Z_{n}(x)\right) W_{n}^{+}\left(\lambda_{n} ; x\right) d \mu(x) \\
& =\int_{C_{a, b}^{\prime}[0, T]} \exp \left\{i(w, y)_{C_{a, b}^{\prime}}^{\sim}\right\} \\
& \quad \times \lim _{n \rightarrow \infty} \exp \left\{-\frac{1}{2 \lambda_{n}} \sum_{j=1}^{n}\left(w, g_{j}\right)_{C_{a, b}^{\prime}}^{2}\right. \\
& \left.\left.+i \lambda_{n}^{-1 / 2} \sum_{j=1}^{n}\left(w, g_{j}\right)_{C_{a, b}^{\prime}} A\right\}\right\} d f(w) \\
& =\int_{C_{a, b}^{\prime}[0, T]} \exp \left\{i(w, y)_{C_{a, b}^{\prime}}^{\sim}\right\} \psi_{P}(-i q ; w) d f(w) .
\end{aligned}
$$

Given $q$ with $|q|>q_{0}$, let the set function $f_{q}^{P}$ : $\mathscr{B}\left(C_{a, b}^{\prime}[0, T]\right) \rightarrow \mathbb{C}$ be given by (62). Then, by the definition of the class $\mathscr{F}_{q_{0}}^{*}$, the inequality (64) holds. From this, we see that $f_{q}^{P}$ belongs to $\mathscr{M}\left(C_{a, b}^{\prime}[0, T]\right)$ and that $\mathscr{P}_{q}(F)$ can be expressed as the third expression of (63) for s-a.e. $y \in$ $C_{a, b}[0, T]$ and all $y \in C_{a, b}^{\prime}[0, T]$. Thus, we conclude the proof of Theorem 15.

In Theorem 16 below, we also establish the existence of the sequential $\mathcal{N}$-function space transform of functionals in $\mathscr{F}^{*}\left(C_{a, b}[0, T]\right)$.

Theorem 16. Let $q_{0}$ and $F \in \mathscr{F}_{q_{0}}^{*}$ be as in Proposition 12. Then for all real $q$ with $|q|>q_{0}$, the sequential $\mathcal{N}$-function space transform of $F, \mathcal{N}_{q}(F)$ exists and is an element of $\mathscr{F}^{*}\left(C_{a, b}[0, T]\right)$ with associated measure $f_{q}^{N}$ defined by

$$
f_{q}^{N}(E)=\int_{E} \psi_{N}(-i q ; w) d f(w), \quad E \in \mathscr{B}\left(C_{a, b}^{\prime}[0, T]\right),
$$

where $\psi_{N}$ is given by (44) and $f$ is the associated measure of $F$ by (36). Furthermore, one sees that for s-a.e. $y \in C_{a, b}[0, T]$ and all $y \in C_{a, b}^{\prime}[0, T]$,

$$
\begin{aligned}
& \mathscr{N}_{q}(F)(y) \\
& =\int_{C_{a, b}^{\prime}[0, T]} \exp \left\{i(w, y)_{C_{a, b}^{\prime}}^{\sim}\right\} \psi_{N}(-i q ; w) d f(w) \\
& =\int_{C_{a, b}^{\prime}[0, T]} \exp \left\{i(w, y)_{C_{a, b}^{\prime}}^{\sim}\right\} d f_{q}^{N}(w)
\end{aligned}
$$

with

$$
\begin{aligned}
\left\|f_{q}^{N}\right\| & <\int_{C_{a, b}^{\prime}[0, T]} \exp \left\{\frac{1}{\sqrt{2 q_{0}}}\|w\|_{C_{a, b}^{\prime}}\|a\|_{C_{a, b}^{\prime}}\right\} d|f|(w) \\
& <+\infty
\end{aligned}
$$

In view of Theorems 15 and 16, we obtain the following assertion.

Theorem 17. Let $q_{0}$ be a positive real number and let $F \in$ $\mathscr{F}_{q_{0} / 4}^{*}$ be given by (36). Then

(i) for all real $q$ with $|q|>q_{0}, \mathscr{P}_{q}(F)$ and $\mathscr{P}_{-q}(F)$ are elements of $\mathscr{F}_{q_{0}}^{*}$, and one has

$$
\mathcal{N}_{-q}\left(\mathscr{P}_{q}(F)\right) \approx F \approx \mathscr{N}_{q}\left(\mathscr{P}_{-q}(F)\right) ;
$$

and

(ii) for all real $q$ with $|q|>q_{0}, \mathcal{N}_{q}(F)$ and $\mathcal{N}_{-q}(F)$ are elements of $\mathscr{F}_{q_{0}}^{*}$, and one has

$$
\mathscr{P}_{-q}\left(\mathcal{N}_{q}(F)\right) \approx F \approx \mathscr{P}_{q}\left(\mathcal{N}_{-q}(F)\right) \text {. }
$$

We finish this section with some examples to apply our results. Let $\mathscr{M}(\mathbb{R})$ be the class of complex-valued, countably additive Borel measures on $\mathscr{B}(\mathbb{R})$, the Borel class of $\mathbb{R}$. For $\tau \in \mathscr{M}(\mathbb{R})$, the Fourier transform $\hat{\tau}$ of $\tau$ is a complex-valued function defined on $\mathbb{R}$ by the formula

$$
\widehat{\tau}(u)=\int_{\mathbb{R}} \exp \{i u v\} d \tau(v) .
$$

Let $h \in C_{a, b}^{\prime}[0, T]$ and let $\tau \in \mathscr{M}(\mathbb{R})$. Define $F_{h, \tau}$ : $C_{a, b}[0, T] \rightarrow \mathbb{C}$ by

$$
F_{h, \tau}(x)=\widehat{\tau}\left((h, x)_{C_{a, b}^{\prime}}^{\sim}\right) .
$$

Define a function $\phi: \mathbb{R} \rightarrow C_{a, b}^{\prime}[0, T]$ by $\phi(v)=v h$ and let $f=\tau \circ \phi^{-1}$. It is quite clear that $f$ is in $\mathscr{M}\left(C_{a, b}^{\prime}[0, T]\right)$ and is supported by $[h]$, the subspace of $C_{a, b}^{\prime}[0, T]$ spanned by $\{h\}$. Now, for s-a.e. $x \in C_{a, b}[0, T]$ and all $x \in C_{a, b}^{\prime}[0, T]$,

$$
\begin{aligned}
F_{h, \tau}(x) & =\int_{\mathbb{R}} \exp \left\{i(h, x)_{C_{a, b}^{\prime}}^{\sim} v\right\} d \tau(v) \\
& =\int_{\mathbb{R}} \exp \left\{i(\phi(v), x)_{C_{a, b}^{\prime}}^{\sim}\right\} d \tau(v) \\
& =\int_{C_{a, b}^{\prime}[0, T]} \exp \left\{i(w, x)_{C_{a, b}^{\prime}}^{\sim}\right\} d\left(\tau \circ \phi^{-1}\right)(w) \\
& =\int_{C_{a, b}^{\prime}[0, T]} \exp \left\{i(w, x)_{C_{a, b}^{\prime}}^{\sim}\right\} d f(w) .
\end{aligned}
$$

Thus, $F_{h, \tau}$ is an element of $\mathscr{F}^{*}\left(C_{a, b}[0, T]\right)$.

Suppose that for a fixed positive real number $q_{0}>0$,

$$
\int_{\mathbb{R}} \exp \left\{\left(2 q_{0}\right)^{-1 / 2}\|h\|_{C_{a, b}^{\prime}}\|a\|_{C_{a, b}^{\prime}}|v|\right\} d|\tau|(v)<+\infty .
$$


It is easy to show that condition (74) is equivalent to condition (45) above. Thus, applying Theorems 15 and 16, the sequential function space transforms $\mathscr{P}_{q}\left(F_{h, \tau}\right)$ and $\mathscr{N}_{q}\left(F_{h, \tau}\right)$ of $F_{h, \tau}$ exist for all $q \in \mathbb{R}$ with $|q|>q_{0}$ if the complex measure $\tau$ corresponding to $F_{h, \tau}$ satisfies condition (74).

Next, we present functionals $F_{h, \tau}$ in $\mathscr{F}^{*}\left(C_{a, b}[0, T]\right)$ whose associated measures satisfy the condition (74).

Example 18. Let $S: C_{a, b}^{\prime}[0, T] \rightarrow C_{a, b}^{\prime}[0, T]$ be the linear operator defined by $S w(t)=\int_{0}^{t} w(s) d b(s)$. Then, the adjoint operator $S^{*}$ of $S$ is given by $S^{*} w(t)=\int_{0}^{t}(w(T)-w(s)) d b(s)$. Using an integration by parts formula, we have

$$
\left(S^{*} b, x\right)_{C_{a, b}^{\prime}}^{\sim}=\int_{0}^{T} x(t) d b(t)
$$

Given real numbers $m$ and $\sigma^{2}$ with $\sigma^{2}>0$, let $\tau_{m, \sigma^{2}}$ be the Gaussian measure given by

$$
\begin{array}{r}
\tau_{m, \sigma^{2}}(B)=\left(2 \pi \sigma^{2}\right)^{-1 / 2} \int_{B} \exp \left\{-\frac{(v-m)^{2}}{2 \sigma^{2}}\right\} d v, \\
B \in \mathscr{B}(\mathbb{R}) .
\end{array}
$$

Then $\tau_{m, \sigma^{2}} \in \mathscr{M}(\mathbb{R})$ and

$$
\begin{aligned}
\widehat{\tau_{m, \sigma^{2}}}(u) & =\int_{\mathbb{R}} \exp \{i u v\} d \tau_{m, \sigma^{2}}(v) \\
& =\exp \left\{-\frac{1}{2} \sigma^{2} u^{2}+i m u\right\}, u \in \mathbb{R} .
\end{aligned}
$$

The complex measure $\tau_{m, \sigma^{2}}$ given by (76) satisfies condition (74) for all real $q_{0}>0$. Thus, we can apply the results in Section 4 to the functional of the form

$$
\begin{aligned}
& F_{h, \tau_{m, \sigma^{2}}}(x) \\
& \quad=\widehat{\tau_{m, \sigma^{2}}}\left((h, x)_{C_{a, b}^{\prime}}^{\sim}\right) \\
& \quad=\exp \left\{-\frac{1}{2} \sigma^{2}\left[(h, x)_{C_{a, b}^{\prime}}^{\sim}\right]^{2}+i m(h, x)_{C_{a, b}^{\prime}}^{\sim}\right\} .
\end{aligned}
$$

We note that $F_{h, \tau_{m, \sigma^{2}}} \in \cup_{q_{0}>0} \mathscr{F}_{q_{0}}^{*}$ and so that for every $q \in \mathbb{R}-\{0\}$, the sequential transforms of $F_{h, \tau_{m, \sigma^{2}}}, \mathscr{P}_{q}\left(F_{h, \tau_{m, \sigma^{2}}}\right)$, and $\mathcal{N}_{q}\left(F_{h, \tau_{m, \sigma^{2}}}\right)$ exist and are elements of $\mathscr{F}\left(C_{a, b}[0, T]\right)$ by Theorems 15 and 16 above. Furthermore, we have that

$$
\mathcal{N}_{-q}\left(\mathscr{P}_{q}\left(F_{h, \tau_{m, \sigma^{2}}}\right)\right) \approx F_{h, \tau}
$$

for all real $q \in \mathbb{R}-\{0\}$ by Theorem 17 . In fact, by a simple calculation, we obtain that for all $q \in \mathbb{R}-\{0\}$,

$$
\begin{aligned}
& \mathscr{P}_{q}\left(F_{h, \tau_{m, \sigma^{2}}}\right)(y) \\
&=\int_{\mathbb{R}} \exp \left\{i(h, y)_{C_{a, b}^{\prime}} v-\frac{i}{2 q}\|h\|_{C_{a, b}^{\prime}}^{2} v^{2}\right. \\
&\left.+i(-i q)^{-1 / 2}(h, a)_{C_{a, b}^{\prime}} v\right\} d \tau_{m, \sigma^{2}}(v),
\end{aligned}
$$

$$
\begin{aligned}
& \mathcal{N}_{-q}\left(F_{h, \tau_{m, \sigma^{2}}}\right)(y) \\
&=\int_{\mathbb{R}} \exp \left\{i(h, y)_{C_{a, b}^{\prime}}^{\sim} v+\frac{i}{2 q}\|h\|_{C_{a, b}^{\prime}}^{2} v^{2}\right. \\
&\left.-i(-i q)^{-1 / 2}(h, a)_{C_{a, b}^{\prime}} v\right\} d \tau_{m, \sigma^{2}}(v)
\end{aligned}
$$

for s-a.e. $y \in C_{a, b}[0, T]$ and all $y \in C_{a, b}^{\prime}[0, T]$.

In particular, if we choose $h=S^{*} b, m=0$, and $\sigma^{2}=2$ in (78), we have

$$
F_{S^{*} b, \tau_{0,2}}(x)=\exp \left\{-\left(\int_{0}^{T} x(t) d b(t)\right)^{2}\right\} .
$$

Example 19. The functional

$$
F_{\delta_{S^{*} b}}(x)=\exp \left\{i \int_{0}^{T} x(t) d b(t)\right\}, \quad x \in C_{a, b}[0, T]
$$

also is a functional under our consideration because

$$
\begin{aligned}
F_{\delta_{S^{*} b}}(x) & =\exp \left\{i\left(S^{*} b, x\right)_{C_{a, b}^{\prime}}^{\sim}\right\} \\
& =\int_{C_{a, b}^{\prime}[0, T]} \exp \left\{i(w, x)_{C_{a, b}^{\prime}}^{\sim}\right\} d \delta_{S^{*} b}(w),
\end{aligned}
$$

where $\delta_{\delta_{S^{*} b}}$ is the Dirac measure concentrated at $S^{*} b$ in $C_{a, b}^{\prime}[0, T]$. The Dirac measure $\delta_{\delta_{S^{*} b}}$ also satisfies condition (45) for all real $q_{0}>0$; that is, $F_{\delta_{S^{*} b}} \in \cup_{q_{0}>0} \mathscr{F}_{q_{0}}^{*}$.

\section{Concluding Remarks}

In this section, we examine certain aspects among the generalized analytic Fourier-Feynman transform $[12,13]$, the generalized integral transform $[15,16]$, and the sequential transforms for functionals on $C_{a, b}[0, T]$. As possible, we adopt the definitions and notation of $[11-13,15,16]$ for the (generalized) analytic Fourier-Feynman transform and the (generalized) integral transform.

A major goal of the authors in $[12,13,15,16]$ was to generalize the concepts of the analytic Fourier-Feynman transform and the integral transform of the functionals of paths for the generalized Brownian motion process.

In $[5,6]$, the authors obtained the existence of the $L_{2}$ analytic Fourier-Feynman transform $T_{q}^{(2)}(F)$ for several large classes of functionals $F$ on Wiener space $C_{0}[0, T]$. In particular, they showed that for all real $q \neq 0$,

$$
T_{-q}^{(2)}\left(T_{q}^{(2)}(F)\right)(y)=F(y)
$$

for s-a.e. $y \in C_{0}[0, T]$. Thus, $L_{2}$ analytic Fourier-Feynman

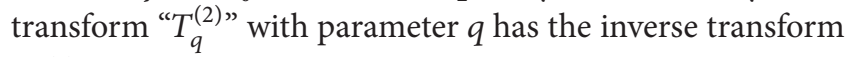
" $T_{-q}^{(2) " . ~ H o w e v e r, ~ i n ~ v i e w ~ o f ~ t h e ~ r e s u l t s ~ i n ~}[12,13],(84)$ does not hold for functionals $F$ on $C_{a, b}[0, T]$. As mentioned in Section 1, the existence of an inverse generalized Fourier-Feynman transform on $C_{a, b}[0, T]$ has not yet been 
established. It is not easy to verify the existence of the inverse generalized Fourier-Feynman transform because the generalized Brownian motion process has a drift term $a(t)$.

In $[9,10]$, the authors studied the integral transform of functionals $F$ in $L_{2}\left(C_{0}[0, T]\right)$. They showed that for $F \in$ $L_{2}\left(C_{0}[0, T]\right)$ and nonzero complex numbers $\alpha$ and $\beta$ with $|\beta| \leq 1, \beta \neq \pm 1, \operatorname{Re}\left(1-\beta^{2}\right)>0, \alpha=\sqrt{1-\beta^{2}}$, and $-\pi / 4<\arg (\alpha)<\pi / 4$,

$$
\mathscr{F}_{\alpha^{\prime}, 1 / \beta} \mathscr{F}_{\alpha, \beta} F(y)=F(y), \quad y \in C_{0}[0, T],
$$

where $\alpha^{\prime}=\sqrt{1-1 / \beta^{2}}$. That is to say, “ $\mathscr{F}_{\alpha, \beta}^{-1}$ " is given by " $\mathscr{F}_{i \alpha / \beta, 1 / \beta}$ ". In [16], Chang et al. presented a version of inverse transform of the generalized integral transform $\mathscr{F}_{\alpha, \beta}$ as follows: for appropriate functionals $F$ on $K_{a, b}[0, T]$, the complexification of $C_{a, b}[0, T]$,

$$
\mathscr{F}_{-i \alpha, 1} \mathscr{F}_{i \alpha, 1} \mathscr{F}_{-\alpha / \beta, 1 / \beta} \mathscr{F}_{\alpha, \beta} F(y)=F(y)
$$

for $y \in K_{a, b}[0, T]$; that is,

$$
\mathscr{F}_{\alpha, \beta}^{-1}=\mathscr{F}_{-i \alpha, 1} \mathscr{F}_{i \alpha, 1} \mathscr{F}_{-\alpha / \beta, 1 / \beta}
$$

But they pointed out that for any nonzero complex numbers $\alpha_{1}, \alpha_{2}, \beta_{1}$ and $\beta_{2}$, there are no nonzero complex numbers $\alpha^{\prime}$, and $\beta^{\prime}$ such that

$$
\mathscr{F}_{\alpha_{1}, \beta_{1}} \mathscr{F}_{\alpha_{2}, \beta_{2}}=\mathscr{F}_{\alpha^{\prime}, \beta^{\prime}} .
$$

Hence, the inverse generalized integral transform, $\mathscr{F}_{\alpha, \beta}^{-1}$, cannot be expressed as a simple integral transform.

Let us return to the singular integral transforms discussed in this paper. Theorem 17 tells us that the sequential function space transforms $\mathscr{P}_{q}$ and $\mathscr{N}_{q}$ have inverse transforms $\mathscr{P}_{q}^{-1}=$ $\mathcal{N}_{-q}$ and $\mathcal{N}_{q}^{-1}=\mathscr{P}_{-q}$, respectively. By choosing $a(t) \equiv 0$ and $b(t)=t$ on $[0, T]$, the function space $C_{a, b}[0, T]$ reduces to the Wiener space $C_{0}[0, T]$ and the sequential transforms $\mathscr{P}_{q}$ and $\mathcal{N}_{q}$ coincide.

Let $F$ be as in Theorem 15. Then, by the definition of the $L_{2}$ generalized analytic Fourier-Feynman transform, we can see that $T_{q}^{(2)}(F)$ of $F$ exists and

$$
T_{q}^{(2)}(F)(y)=\mathscr{P}_{q}(F)(y)
$$

for s-a.e. $y \in C_{a, b}[0, T]$ and all $y \in C_{a, b}^{\prime}[0, T]$. This fact tells us that the sequential $\mathcal{N}$-function space transform $\mathcal{N}_{-q}$ plays a prominent role as an inverse transform of the generalized analytic Fourier-Feynman transform $T_{q}^{(2)}$.

\section{Acknowledgments}

The authors would like to express their gratitude to the referees for their valuable comments and suggestions which have improved the original paper. This research was supported by Basic Science Research Program through the National Research Foundation of Korea (NRF) funded by the Ministry of Education (2011-0014552) and Basic Science Research
Program through the National Research Foundation of Korea (NRF) funded by the Ministry of Science, ICT \& Future Planning (2012R1A1A1004774). The authors declare that there is no conflict of interests regarding the publication of this paper.

\section{References}

[1] R. H. Cameron, "Some examples of Fourier-Wiener transforms of analytic functionals," Duke Mathematical Journal, vol. 12, no. 3, pp. 485-488, 1945.

[2] R. H. Cameron and W. T. Martin, "Fourier-Wiener transforms of analytic functionals," Duke Mathematical Journal, vol. 12, pp. 489-507, 1945.

[3] R. H. Cameron and W. T. Martin, "Fourier-Wiener transforms of functionals belonging to $L_{2}$ over the space C," Duke Mathematical Journal, vol. 14, no. 1, pp. 99-107, 1947.

[4] M. D. Brue, A functional transform for Feynman integrals similar to the Fourier transform [Thesis], University of Minnesota, St. Paul, Minn, USA, 1972.

[5] R. H. Cameron and D. A. Storvick, "An $L_{2}$ analytic FourierFeynman transform," The Michigan Mathematical Journal, vol. 23, no. 1, pp. 1-30, 1976.

[6] G. W. Johnson and D. L. Skoug, "An $L_{p}$ analytic FourierFeynman transform," The Michigan Mathematical Journal, vol. 26, no. 1, pp. 103-127, 1979.

[7] Y.-J. Lee, "Integral transforms of analytic functions on abstract Wiener spaces," Journal of Functional Analysis, vol. 47, no. 2, pp. 153-164, 1982.

[8] Y.-J. Lee, "Unitary operators on the space of $L^{2}$-functions over abstract Wiener spaces," Soochow Journal of Mathematics, vol. 13, no. 2, pp. 165-174, 1987.

[9] B. S. Kim and D. Skoug, "Integral transforms of functionals in $L_{2}\left(C_{0}[0, T]\right)$," The Rocky Mountain Journal of Mathematics, vol. 33, no. 4, pp. 1379-1393, 2003.

[10] S. J. Chang, H. S. Chung, and D. Skoug, "Convolution products, integral transforms and inverse integral transforms of functionals in $L_{2}\left(C_{0}[0, T]\right)$," Integral Transforms and Special Functions, vol. 21, no. 1-2, pp. 143-151, 2010.

[11] D. Skoug and D. Storvick, "A survey of results involving transforms and convolutions in function space," The Rocky Mountain Journal of Mathematics, vol. 34, no. 3, pp. 1147-1175, 2004.

[12] S. J. Chang and D. Skoug, "Generalized Fourier-Feynman transforms and a first variation on function space," Integral Transforms and Special Functions, vol. 14, no. 5, pp. 375-393, 2003.

[13] S. J. Chang, J. G. Choi, and D. Skoug, "Integration by parts formulas involving generalized Fourier-Feynman transforms on function space," Transactions of the American Mathematical Society, vol. 355, no. 7, pp. 2925-2948, 2003.

[14] S. J. Chang, J. G. Choi, and D. Skoug, "Generalized FourierFeynman transforms, convolution products, and first variations on function space," The Rocky Mountain Journal of Mathematics, vol. 40, no. 3, pp. 761-788, 2010.

[15] S. J. Chang, H. S. Chung, and D. Skoug, "Integral transforms of functionals in $L^{2}\left(C_{a, b}[0, T]\right)$," The Journal of Fourier Analysis and Applications, vol. 15, no. 4, pp. 441-462, 2009.

[16] S. J. Chang, H. S. Chung, and D. Skoug, "Some basic relationships among transforms, convolution products, first variations 
and inverse transforms," Central European Journal of Mathematics, vol. 11, no. 3, pp. 538-551, 2013.

[17] J. Yeh, "Singularity of Gaussian measures on function spaces induced by Brownian motion processes with non-stationary increments," Illinois Journal of Mathematics, vol. 15, pp. 37-46, 1971.

[18] J. Yeh, Stochastic Processes and the Wiener Integral, Marcel Dekker, New York, NY, USA, 1973.

[19] S. J. Chang and D. M. Chung, "Conditional function space integrals with applications," The Rocky Mountain Journal of Mathematics, vol. 26, no. 1, pp. 37-62, 1996.

[20] L. A. Shepp, "Radon-Nikodym derivatives of Gaussian measures," Annals of Mathematical Statistics, vol. 37, pp. 321-354, 1966.

[21] R. H. Cameron and D. A. Storvick, A Simple Definition of the Feynman Integral With Applications, vol. 46 of no. 288 of Memoirs of the American Mathematical Society, American Mathematical Society, Providence, RI, USA, 1983.

[22] R. P. Feynman, "Space-time approach to non-relativistic quantum mechanics," Reviews of Modern Physics, vol. 20, pp. 367-387, 1948.

[23] R. H. Cameron, "A family of integrals serving to connect the Wiener and Feynman integrals," Journal of Mathematics and Physics, vol. 39, pp. 126-140, 1960. 


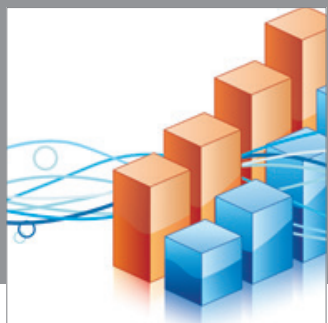

Advances in

Operations Research

mansans

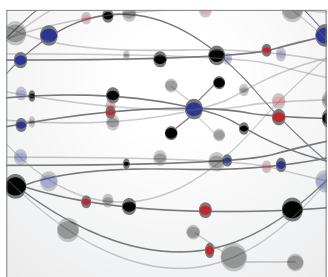

The Scientific World Journal
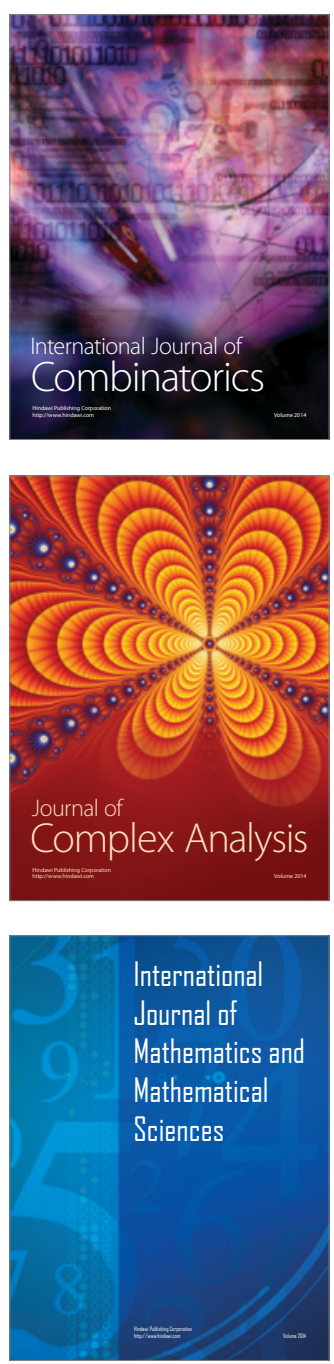
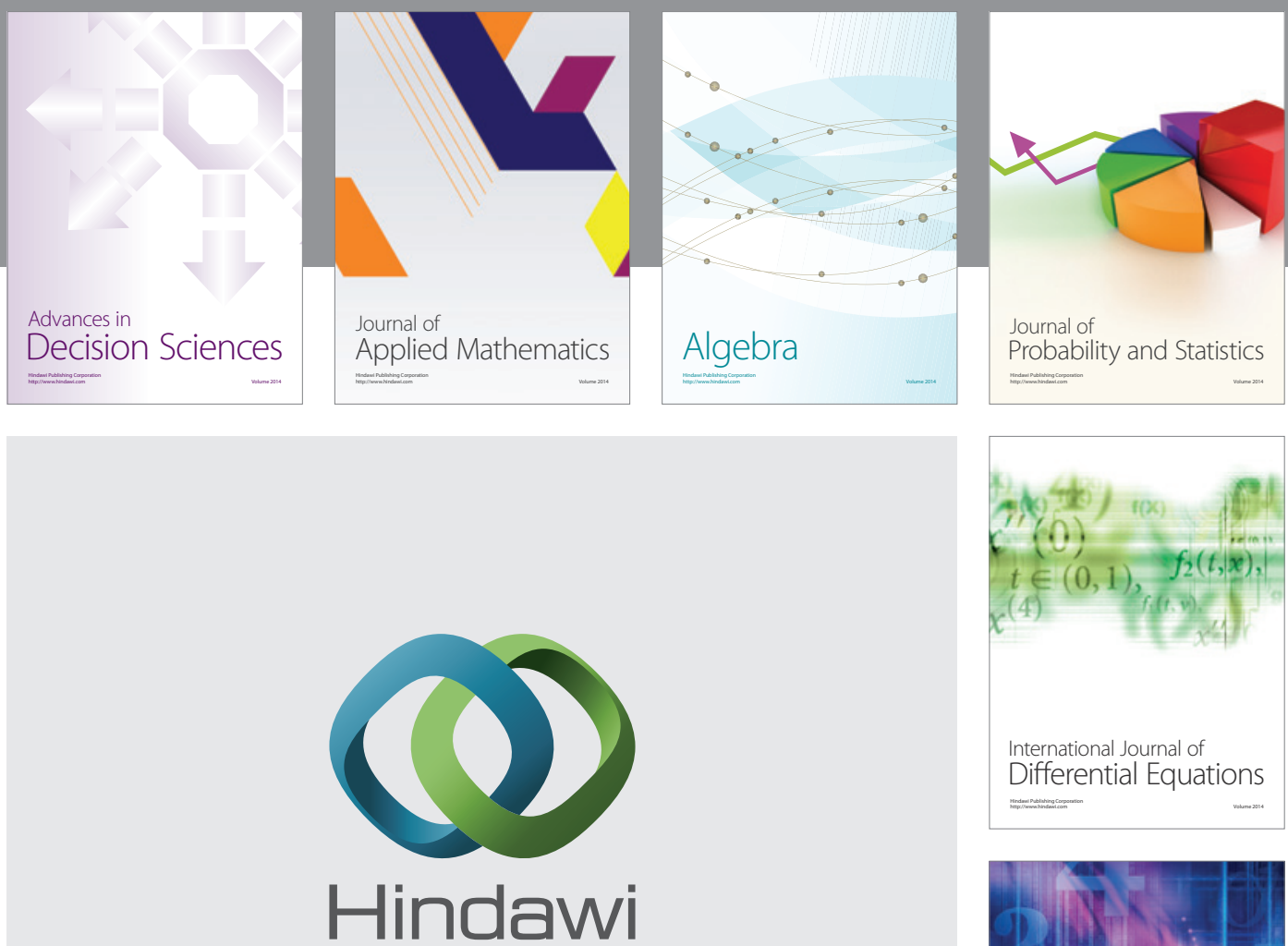

Submit your manuscripts at http://www.hindawi.com
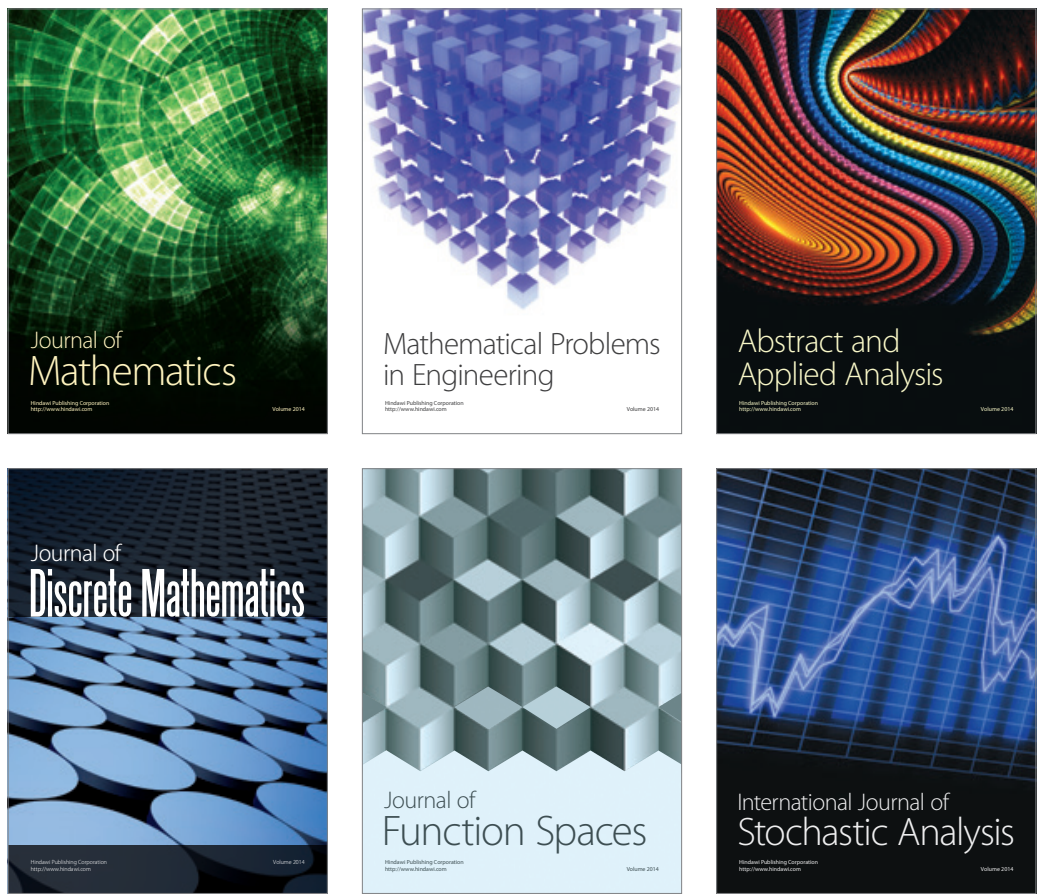

Journal of

Function Spaces

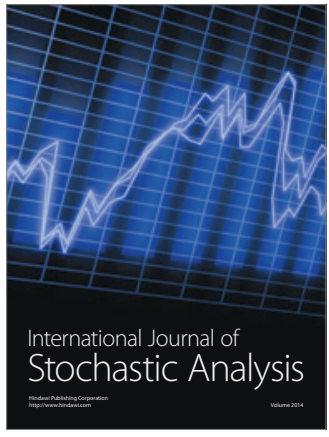

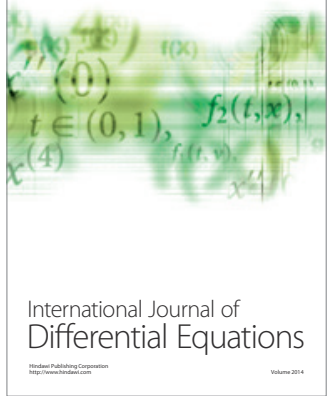
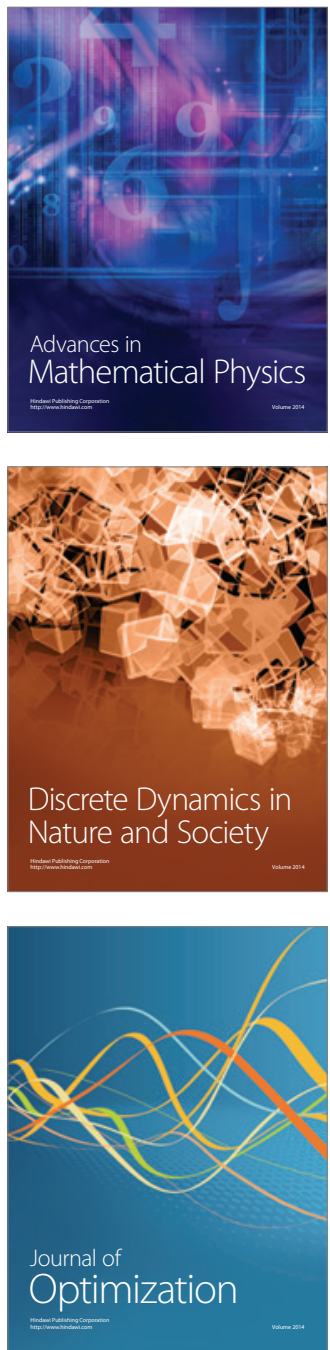